\title{
Study of Patients with Bilateral Knee Osteoarthritis Undergoing Total Knee Replacement Procedure with Coexisting Lumbar Spondylosis Symptoms
}

\author{
Sanjay Bhalchandra Londhe ${ }^{1}$, Ravi Vinod Shah ${ }^{2}$, Meghana Patwardhan ${ }^{3}$, Amit Pankaj Doshi ${ }^{4}$, \\ Shubhankar Sanjay Londhe ${ }^{5}$, Kavita Subhedar ${ }^{6}$, Vishal Kundnani ${ }^{7}$, Jwalant Patel ${ }^{7}$ \\ ${ }^{1}$ Department of Orthopedic Surgery, Holy Spirit Hospital, Mumbai, India \\ ${ }^{2}$ Department of Orthopedic Surgery, Criticare Hospital, Mumbai, India \\ ${ }^{3}$ Department of Pain Medicine and Anesthesia, Criticare Hospital, Mumbai, India \\ ${ }^{4}$ Department of Product Management, Meril Lifesciences, Mumbai, India \\ ${ }^{5}$ Department of Computer Science, The Vishwanath Karad MIT World Peace University, Pune, India \\ ${ }^{6}$ Department of Clinical Data Management, Criticare Hospital, Mumbai, India \\ ${ }^{7}$ Division of Spine Surgery, Department of Orthopaedics, Bombay Hospital, Mumbai, India
}

Study Design: This is a prospective cohort study involving patients who were followed for 2 years after total knee replacement (TKR) to determine changes in lumbar spine and knee symptoms.

Purpose: The objectives of this study were to determine the percentage of patients undergoing bilateral TKR who present with coexisting lumbar spine problems and determine if TKR relieves lumbar spine symptoms.

Overview of Literature: No studies quantify the percentage of TKR patients who experience relief of lumbar spine symptoms after TKR surgery.

Methods: The study included 200 patients (164 females, 36 males) undergoing primary TKR. Follow-up was performed at 4 weeks, 3 , 6, 12, and 24 months. Lumbar spine and knee symptom improvements were assessed using the Oswestry Disability Index (ODI) and Oxford Knee Score, respectively.

Results: All 200 patients undergoing bilateral TKR presented with radiographic lumbar spine degenerative pathology; 60\% ( $n=120)$ of the patients presented with moderate to severe clinical symptoms of lumbar spondylosis, including $54 \%$ ( $n=108$ ) with degenerative lumbar spondylosis and lumbar canal stenosis and $6 \%(n=12)$ with degenerative spondylolisthesis. Of the 120 patients who presented with lumbar spine problems, $90 \%$ ( $n=108$ ) reported improvement in their symptoms; the ODI score improved from $42.5 \% \pm 4.1 \%$ preoperative score to $15.6 \% \pm 2.3 \%$ postoperative score ( $p$-value $<0.001$ ). Of the 12 patients with no improvement, 10 patients underwent percutaneous procedures for their lumbar spine pathology with good results, one patient underwent surgery, and one declined any intervention.

Conclusions: A significant number of patients $(60 \%)$ undergoing bilateral TKR also present with symptomatic lumbar spine problems. Patients with mild to moderate lumbar spine degenerative symptoms and no associated severe radiating pain on activity are more likely to experience relief of their symptoms post-TKR.

Keywords: Lumbar disc degeneration; Osteoarthritis; Total knee replacement

Received Jun 4, 2020; Revised Aug 10, 2020; Accepted Aug 31, 2020

Corresponding author: Sanjay Bhalchandra Londhe

Department of Orthopedic Surgery, Holy Spirit Hospital, Mahakali Caves Road, Andheri East, Mumbai 400093, India

Tel: +91-2228239205, Fax: +91-2228239205, E-mail: sanlondhe@yahoo.com 


\section{Introduction}

Total Knee Replacement (TKR) is one of the most successful surgeries in modern-day orthopedics. The number of TKRs is rising around the world every year. The rise in TKR procedures is partially due to the worldwide increase in life expectancy, leading to a rise in the incidence of degenerative diseases. TKR surgery leads to improvements in pain, physical functioning, and quality of life in patients with severe osteoarthritis (OA) [1-6]. These patients often present with comorbidities, such as diabetes, hypertension, and chronic kidney disease. Knee OA and lumbar spine degenerative are common comorbidities in the elderly population [7-9]. Lumbar degenerative disease presents as lower back pain and/or radiating pain to the legs at rest or during activity. Patients undergoing TKR for advanced OA of the knee often exhibit these symptoms of coexisting lumbar spondylosis, such as mechanical lower back pain or radiating pain to the lower limbs $[10,11]$. An orthopedic surgeon's dilemma is what to address first, the knee OA with TKR or the lumbar spondylosis. To the best of our knowledge, no supporting research exists to answer this question. The association of coexisting lumbar spine symptoms aggravates the overall symptoms and function in patients with advanced knee OA undergoing TKR. Moreover, the persistence of coexisting lumbar spine symptoms after TKR may adversely affect postoperative outcomes in terms of pain and function, even after a technically well-executed TKR [11-13]. Chang et al. [14] studied the prevalence and severity of coexisting lumbar spondylosis in terms of radiographic lumbar spine degeneration and lumbar spine symptoms in patients with advanced knee OA undergoing TKR. They also studied the effects of coexisting lumbar spondylosis on clinical scores for pre- and post-TKR assessments and pre- and postoperative quality of life before and 2 years after TKR. In the study of Chang et al. [14], 51\% of patients undergoing TKR had at least one moderate to severe lumbar spine symptom and patients with severe radicular pain on activity before the TKR were likely to demonstrate poor knee function 2 years post-TKR. To the best of our knowledge, no studies demonstrate the effects of TKR surgery on the improvement of coexisting lumbar spondylosis symptoms after surgery. The aim of this study was to determine the percentage of patients undergoing TKR surgery that present with lumbar spine problems and to determine if TKR improves lumbar spine symptoms.

\section{Materials and Methods}

\section{Patients}

We conducted a prospective cohort study on 200 patients with knee OA undergoing primary TKR between January 2015 and December 2016 at Holy Spirit Hospital and Criticare Hospital. One hundred and sixty-four female and 36 male patients were present in the study. The mean age was $64.5 \pm 14.6$ years for females and $71.1 \pm 14.3$ years for males. The body mass index (BMI) was $29.3 \pm 6.1 \mathrm{~kg} /$ $\mathrm{m}^{2}$ for females and $28.7 \pm 5.9 \mathrm{~kg} / \mathrm{m}^{2}$ for males (Table 1). The local ethics committee approval and informed consent were obtained for all patients. All patients undergoing TKR received anteroposterior (AP) and lateral view $\mathrm{X}$-rays of their lumbosacral spine as part of the preoperative screening. The radiograph covered the area from thoracic 7 (T7) to sacrum 2 (S2). Radiographs were digitally

Table 1. Patient characteristics undergoing bilateral TKR

\begin{tabular}{|c|c|c|c|}
\hline Characteristic & Females & Males & $p$-value \\
\hline No. of patients & 164 & 36 & \\
\hline Age (yr) & $64.5 \pm 14.6(58-78)$ & $71.1 \pm 14.3(61-82)$ & 0.0146 \\
\hline Body mass index $\left(\mathrm{kg} / \mathrm{m}^{2}\right)$ & $29.3 \pm 6.1(18.7-42.9)$ & $28.7 \pm 5.9(18.1-41.4)$ & 0.5915 \\
\hline Simultaneous bilateral TKR & $130(79.26)$ & $28(77.77)$ & 0.8429 \\
\hline Staged bilateral TKR & $34(20.73)$ & $18(22.22)$ & 0.8428 \\
\hline Hospital stay duration (day) & $4.6 \pm 0.6$ & $4.5 \pm 0.5$ & 0.3530 \\
\hline Postoperative knee alignment $\left({ }^{\circ}\right)^{\text {al }}$ & $1.6 \pm 0.3$ & $1.5 \pm 0.5$ & 0.1158 \\
\hline
\end{tabular}

Values are presented as mean $\pm S D$ (range), number $(\%)$, or mean $\pm S D$, unless otherwise stated.

TKR, total knee replacement; SD, standard deviation.

alHip-knee-ankle angle or femorotibial angel. 
acquired, and assessments were made on a 60.96-cm (24inch) LCD (liquid crystal display) monitor. The degree of lumbar spine degeneration was radiographically assessed using a validated radiographic grading system described by Wilke et al. [15]. The grading system includes the following variables: height loss of disk space, osteophyte formation, and diffuse endplate sclerosis. Each of these variables was graded on the AP and lateral projection Xrays. The grade of degeneration was a score based on a 4-point scale: 0 , no degeneration; 1 , mild degeneration; 2 , moderate degeneration; and 3, severe degeneration. Unilateral TKR patients received spinal anesthesia plus femoral/adductor canal blocks. Simultaneous bilateral TKR patients received continuous spinal epidural anesthesia. The preoperative symptoms of lumbar spondylosis were evaluated using a self-assessment questionnaire, based on the format of Chang et al. [14] (Fig. 1) and the Oswestry Disability Index (ODI). The patients were asked about their lower back pain, radiating pain at rest, and radiating pain on activity. The severity of pain was rated using a Visual Analog Scale (VAS), with 0 representing no pain and 10 representing the worst pain. The lumbar spondylosis symptoms were graded as follows: no/minimal pain, $0-3$

Patient Self Addressed Questionnaire

In the last 4 weeks whether you have experienced lower back pain: yes/no

If yes how would you grade it on scale of 0-10: ( )

Radiating pain on rest (pain starts from the buttock or inguinal area and spreads down to the knee, ankle, or foot or pain starts from the thigh and spreads down to the ankle or foot during rest (sitting, lying down, etc.): yes/no

If yes how would you grade it on scale of $0-10:(\quad$ )

Radiating pain on activity (pain starts from the buttock or inguinal area and spreads down to the knee, ankle, or foot or pain starts from the thigh and spreads down to the ankle or foot during walking, standing, climbing the stairs, etc.: yes/no

Fig. 1. Specimen of Patient Self Addressed Questionnaire.
VAS; moderate pain, 4-6 VAS; and severe pain, 7-10 VAS $[16,17]$. If deemed necessary by a spine specialist, an MRI scan of the lumbosacral spine was obtained. The knee symptoms were assessed preoperatively using the Oxford Knee Score (OKS). The indication for TKR was advanced OA of the knee joint (grade 4, bone on bone) affecting the quality of life and day to day activities. Patients undergoing TKR for rheumatoid arthritis or post-traumatic arthritis were excluded from this study. All patients were operated by a single surgeon and assistant. TKR was performed in a standard fashion with a tourniquet. Patients were followed up at 4 weeks, 3 months, 6 months, 12 months, and 2 years after TKR surgery. Improvement in knee symptoms was assessed with the OKS, while the lumbar spine symptoms were assessed with ODI and VAS. An ODI score $<20$ and VAS score $<3$ was the cut-off point for improvement in lumbar spine symptoms.

\section{Statistical methods}

Pre- and postoperative OKS and ODI were compared using $T$-tests for two means. Data are presented as mean \pm standard deviation, unless otherwise specified. A $p$-value $<0.05$ was considered statistically significant.

\section{Results}

All 200 patients undergoing bilateral TKR presented with radiographic lumbar spine degenerative pathology. This finding is consistent with the findings of Chang et al. [14]. Mild degeneration was found in 13\%, moderate degeneration in $71 \%$, and severe degeneration in $16 \%$ of patients. The mean VAS score for the lower lumbar back pain group was 5.6, for the radiating pain at rest group was 6.3, and for the radiating pain on activity group was 7.1. Based on the definition of clinical grade lumbar spine symptoms by Chang et al. [14], $60 \%$ of the patients presented with moderate/severe symptoms of lumbar spondylosis and degenerative lumbar spine disease (Table 2). Of these patients, 54\% ( $\mathrm{n}=108)$ presented with degenerative lumbar spondylosis and lumbar canal stenosis, and 6\% $(\mathrm{n}=12)$ presented with degenerative spondylolisthesis. Patients were evaluated by an independent examiner 2 years after their TKR surgery. All 200 patients showed improvement in their OKS from a preoperative mean of $20.4 \pm 6.8$ to a postoperative mean of $37.5 \pm 4.2(p<0.001)$ (Table 3$)$. Of the 120 patients who presented with lumbar spine symp- 
toms, $90 \%$ (108 patients) reported improvement in their lumbar spine symptoms. The ODI score improved from

Table 2. Radiographic grading of lumbar spine degeneration, VAS scores, and grade of lumbar spine symptoms (total patients=200)

\begin{tabular}{lc} 
Variable & Value \\
\hline Radiographic lumbar spine degeneration & 0 \\
\hline None & 13 \\
\hline Mild degeneration & 71 \\
\hline Moderate degeneration & 16 \\
\hline Severe degeneration & \\
\hline Lumbar spine symptoms & \\
\hline Lower back pain & $116(58)$ \\
\hline No/mild pain (VAS 0-3) & $56(28)$ \\
\hline Moderate pain (VAS 4-6) & $28(14)$ \\
\hline Severe pain (VAS 7-10) & $152(76)$ \\
\hline Radiating pain at rest & $32(16)$ \\
\hline No/mild pain (VAS 0-3) & $16(8)$ \\
\hline Moderate pain (VAS 4-6) & \\
\hline Severe pain (VAS 7-10) & $124(62)$ \\
\hline Radiating pain on activity & $46(23)$ \\
\hline No/mild pain (VAS 3.1 \pm 2.7$)$ & $30(15)$ \\
\hline Moderate pain (VAS 3.1 \pm 2.4$)$ & \\
\hline Severe pain (VAS 3.0 \pm .9$)$ & \\
\hline
\end{tabular}

Values are presented as $\%$ or number (\%).

VAS, Visual Analog Scale.

Table 3. Improvement in the Oxford Knee Score after TKR

\begin{tabular}{lcc} 
Oxford Knee Score & Mean \pm standard deviation & $p$-value \\
Pre-TKR & $20.4 \pm 6.8$ & $<0.001$ \\
Post-TKR & $37.5 \pm 4.2$ & $<0.001$ \\
\hline
\end{tabular}

TKR, total knee replacement. a preoperative mean of $42.5 \% \pm 4.1 \%$ to a postoperative mean of $15.6 \% \pm 2.3 \%(p<0.001)$. The mean VAS score of $1.9 \pm 0.6$ at 2 years after the TKR was lower compared to the preoperative mean VAS score of $7.1 \pm 1.1(p<0.0001)$. In patients with lumbar canal stenosis, the ODI improved from $42.1 \% \pm 3.8 \%$ to $15.4 \% \pm 1.9 \%(p<0.001) 2$ years after surgery, and the ODI scores in the degenerative spondylolisthesis group improved from $46.1 \% \pm 4.4 \%$ to $17.4 \% \pm 2.6 \%$ $(p<0.001)$ (Table 4). Of the 30 patients who presented with severe radiating pain on activity prior to TKR, 18 patients improved, and 12 patients did not improve. The 18 patients who showed improvement presented with mild/ moderate lumbar spine degeneration and severe radiating pain. The 12 patients (10\%) who did not improve presented with both severe radiating pain on activity and severe lumbar degeneration and lumbar spine pain prior to TKR. In these 12 patients, the ODI only marginally improved from $43.6 \% \pm 4.8 \%$ to $41.4 \% \pm 3.5 \%$, which was not statistically significant $(p=0.213)$. The VAS scores also did not improve in these 12 patients; the mean preoperative VAS score was $7.5 \pm 1.3$, and the 2-year postoperative VAS score was $6.9 \pm 1.5$ ( $p=0.3064)$ (Table 5). Out of these 12 patients, 10 patients underwent percutaneous procedures for their lumbar spine pathology with good results. The percutaneous procedure was in the form of a transforaminal nerve root block with or without interlaminar epidural steroid injection. The percutaneous procedure was carried out with fluoroscopy assistance by a consulting pain specialist as a day procedure. Patients experienced significant relief of their radicular symptoms, even at the 12-month followup. The mean VAS score 12 months after the procedure was 1.6, compared to the mean pre-procedure VAS score of 7.2. Of the two patients whose symptoms persisted even after 2 years post-TKR, one patient underwent lumbar spine decompression (central canal and lateral recess), transforaminal lumbar interbody fusion, and stabilization

Table 4. Improvement in the ODI in post-total knee replacement improved patients $(n=108)$

\begin{tabular}{|c|c|c|c|}
\hline \multirow{2}{*}{ Variable } & \multicolumn{2}{|c|}{ ODI } & \multirow{2}{*}{$p$-value } \\
\hline & Preoperative & Postoperative & \\
\hline Improved symptomatic patients $(n=108)$ & $42.5 \pm 4.1$ & $15.6 \pm 2.3$ & $<0.001$ \\
\hline Improved lumbar canal stenosis patients ( $n=97$ ) & $42.1 \pm 3.8$ & $15.4 \pm 1.9$ & $<0.001$ \\
\hline Improved lumbar degenerative spondylolisthesis patients $(n=11)$ & $46.1 \pm 4.4$ & $17.4 \pm 2.6$ & $<0.001$ \\
\hline
\end{tabular}

Values are presented as mean \pm standard deviation.

ODI, Oswestry Disability Index. 
Table 5. ODI and VAS score in patients whose lumbar spine symptoms did not improve post-total knee replacement $(\mathrm{n}=12)$

\begin{tabular}{lccc} 
Variable & Preoperative & Postoperative & $p$-value \\
ODI & $43.8 \pm 4.8$ & $41.4 \pm 3.5$ & 0.213 \\
VAS & $7.5 \pm 1.3$ & $6.9 \pm 1.5$ & 0.3064 \\
\hline
\end{tabular}

Values are presented as mean \pm standard deviation.

ODI, Oswestry Disability Index; VAS, Visual Analog Scale.

with pedicle screws. The remaining patient declined any surgical intervention.

\section{Discussion}

TKR is a highly successful operation for treating patients with advanced knee OA. TKR helps relieve pain and improve physical functioning, quality of life, and activities of daily living [1-6]. Patients undergoing TKR are usually of advanced age. Consequently, the patients often present with comorbidities like diabetes, hypertension, and chronic renal failure, as well as lumbar spine degenerative symptoms. In our study, $90 \%$ of patients were more than 60 years of age. The percentage of patients undergoing TKR who exhibit lumbar spondylosis symptoms and improvement in lumbar spine symptoms after a successful TKR operation is very important. This information is extremely useful to the operating surgeon for a realistic consultation with the patient before planning a TKR surgery. In our study, $90 \%$ of the 120 patients (108 patients) who presented with moderate/severe lumbar spine symptoms reported improvement in their lumbar spine symptoms after the TKR. Only $10 \%$ of patients $(n=12)$ did not show improvement in symptoms. Ten percent of the patients whose lumbar spine symptoms did not improve post-TKR exhibited a combination of both severe radiating pain on activity and severe lumbar degeneration and lumbar spine pain prior to TKR.

The present study exhibits certain limitations. One of the major limitations of the study is the selection bias toward patients with advanced OA of the knee rather than degenerative disease of the lumbar spine. We are aware of the fact that patients present with different severities of knee and lumbar spine degenerative pathology. The patients coming to the joint clinic exhibit more knee degeneration than lumbar spine degeneration. Patients presenting with more severe degenerative lumbar spine problems than knee problems are more likely to seek help from a spine specialist. Thus, it is entirely possible that patients with more severe degenerative pathology of the lumbar spine were not captured in this study. A similar study by spine specialists or combined studies including both spine and joint surgeons would be useful to contradict the selection bias. To the best of our knowledge, no studies address these confounding issues. The second limitation is the female preponderance (82\%) in our study. This is not unusual; women are more prone to advanced symptomatic OA $[7,18,19]$. The third limitation is that the improvement in lumbar spine symptoms after TKR may be because of improvements in the gait pattern postTKR and improvement in the knee function [20]. Part of the improvement might also be because of the use of pain medicines after the TKR surgery, namely Cox 2 inhibitors and pregabalin [21] and postoperative physiotherapy and rehabilitation, which might improve lumbar spine symptoms.

\section{Conclusions}

The number of patients undergoing TKR is rising daily and a significant percentage of these patients present with associated lumbar spine problems. Orthopedic surgeons face the dilemma of which issue to address first, the knee OA or the lumbar spine pathology. Only one study by Chang et al. [14] addressed this dilemma by investigating the percentage of patients undergoing TKR for severe OA of the knee who have coexisting radiological and symptomatic lumbar spondylosis. To the best of our knowledge, no studies quantify the percentage of TKR patients who experience relief of their lumbar spine symptoms after a successful TKR operation. Our study shows that a significant number of patients with bilateral knee OA (60\%) present with associated lumbar spine degenerative pathology. Patients with mild/moderate lumbar spine degenerative symptoms and no associated severe radiating pain on activity are more likely to experience relief of their symptoms post-TKR. For patients with severe degeneration of the knee and mild to moderate degeneration of the lumbar spine without severe radicular symptoms, we recommend that the knee OA be addressed first. Conversely, patients with severe degeneration of the lumbar spine with associated radicular symptoms should be referred and managed by a spine specialist first. This is extremely important because patients who exhibit severe radicular pain on activity prior to the TKR are likely to demonstrate 
poor knee function 2 years post-TKR surgery.

\section{Conflict of Interest}

No potential conflict of interest relevant to this article was reported.

\section{References}

1. Fitzgerald JD, Orav EJ, Lee TH, et al. Patient quality of life during the 12 months following joint replacement surgery. Arthritis Rheum 2004;51:100-9.

2. Meding JB, Meding LK, Ritter MA, Keating EM. Pain relief and functional improvement remain 20 years after knee arthroplasty. Clin Orthop Relat Res 2012;470:144-9.

3. Vina ER, Hannon MJ, Kwoh CK. Improvement following total knee replacement surgery: exploring preoperative symptoms and change in preoperative symptoms. Semin Arthritis Rheum 2016;45:547-55.

4. Zhang W, Moskowitz RW, Nuki G, et al. OARSI recommendations for the management of hip and knee osteoarthritis, part II: OARSI evidence-based, expert consensus guidelines. Osteoarthritis Cartilage 2008;16:137-62.

5. Skou ST, Roos EM, Laursen MB, et al. A randomized, controlled trial of total knee replacement. N Engl J Med 2015;373:1597-606.

6. Liang MH, Cullen KE, Larson MG, et al. Cost-effectiveness of total joint arthroplasty in osteoarthritis. Arthritis Rheum 1986;29:937-43.

7. Felson DT, Naimark A, Anderson J, Kazis L, Castelli W, Meenan RF. The prevalence of knee osteoarthritis in the elderly: the Framingham Osteoarthritis Study. Arthritis Rheum 1987;30:914-8.

8. Hicks GE, Morone N, Weiner DK. Degenerative lumbar disc and facet disease in older adults: prevalence and clinical correlates. Spine (Phila Pa 1976) 2009;34:1301-6.

9. Reginster JY. The prevalence and burden of arthritis. Rheumatology (Oxford) 2002;41 Supp 1:3-6.

10. Hassett G, Hart DJ, Doyle DV, March L, Spector TD. The relation between progressive osteoarthritis of the knee and long term progression of osteoarthritis of the hand, hip, and lumbar spine. Ann Rheum Dis 2006;65:623-8.

11. McNamara MJ, Barrett KG, Christie MJ, Spengler DM. Lumbar spinal stenosis and lower extremity arthroplasty. J Arthroplasty 1993;8:273-7.

12. Bohl WR, Steffee AD. Lumbar spinal stenosis: a cause of continued pain and disability in patients after total hip arthroplasty. Spine (Phila Pa 1976) 1979;4:16873.

13. Fogel GR, Esses SI. Hip spine syndrome: management of coexisting radiculopathy and arthritis of the lower extremity. Spine J 2003;3:238-41.

14. Chang CB, Park KW, Kang YG, Kim TK. Coexisting lumbar spondylosis in patients undergoing TKA: how common and how serious? Clin Orthop Relat Res 2014;472:710-7.

15. Wilke HJ, Rohlmann F, Neidlinger-Wilke C, Werner K, Claes L, Kettler A. Validity and interobserver agreement of a new radiographic grading system for intervertebral disc degeneration: part I. Lumbar spine. Eur Spine J 2006;15:720-30.

16. Kapstad H, Hanestad BR, Langeland N, Rustoen T, Stavem K. Cutpoints for mild, moderate and severe pain in patients with osteoarthritis of the hip or knee ready for joint replacement surgery. BMC Musculoskelet Disord 2008;9:55.

17. Zelman DC, Dukes E, Brandenburg N, Bostrom A, Gore M. Identification of cut-points for mild, moderate and severe pain due to diabetic peripheral neuropathy. Pain 2005;115:29-36.

18. Crowninshield RD, Rosenberg AG, Sporer SM. Changing demographics of patients with total joint replacement. Clin Orthop Relat Res 2006;443:266-72.

19. Sowers M. Epidemiology of risk factors for osteoarthritis: systemic factors. Curr Opin Rheumatol 2001;13:447-51.

20. Harato K, Nagura T, Matsumoto H, Otani T, Toyama Y, Suda Y. A gait analysis of simulated knee flexion contracture to elucidate knee-spine syndrome. Gait Posture 2008;28:687-92.

21. Romano CL, Romano D, Bonora C, Mineo G. Pregabalin, celecoxib, and their combination for treatment of chronic low-back pain. J Orthop Traumatol 2009;10:185-91. 